Virginia Commonwealth University

vCU Scholars Compass

\title{
Localized surface plasmon resonance enhanced magneto-optical activity in core-shell Fe-Ag nanoparticles
}

L. Wang

College of William and Mary, lwang02@wm.edu

K. Yang

College of William and Mary

C. Clavero

College of William and Mary

See next page for additional authors

Follow this and additional works at: http://scholarscompass.vcu.edu/chem_pubs

Part of the Chemistry Commons

Wang, L., Yang, K., \& Clavero, C., et al. Localized surface plasmon resonance enhanced magneto-optical activity in coreshell Fe-Ag nanoparticles. Journal of Applied Physics, 107, 09B303 (2010). Copyright ( 2010 American Institute of Physics.

\section{Downloaded from}

http://scholarscompass.vcu.edu/chem_pubs/27

This Article is brought to you for free and open access by the Dept. of Chemistry at VCU Scholars Compass. It has been accepted for inclusion in Chemistry Publications by an authorized administrator of VCU Scholars Compass. For more information, please contact libcompass@vcu.edu. 
Authors

L. Wang, K. Yang, C. Clavero, A. J. Nelson, K. J. Carroll, E. E. Carpenter, and R. A. Lukaszew 


\title{
Localized surface plasmon resonance enhanced magneto-optical activity in core-shell Fe-Ag nanoparticles
}

\author{
L. Wang, ${ }^{1, a)}$ K. Yang, ${ }^{2}$ C. Clavero, ${ }^{2}$ A. J. Nelson, ${ }^{1}$ K. J. Carroll, ${ }^{3}$ E. E. Carpenter, ${ }^{3}$ and \\ R. A. Lukaszew ${ }^{1,2}$ \\ ${ }^{1}$ Department of Physics, College of William and Mary, Williamsburg, Virginia 23187, USA \\ ${ }^{2}$ Department of Applied Science, College of William and Mary, Williamsburg, Virginia 23187, USA \\ ${ }^{3}$ Department of Chemistry, Virginia Commonwealth University, Richmond, Virginia 23284, USA
}

(Presented 20 January 2010; received 29 October 2009; accepted 7 December 2009; published online 20 April 2010)

\begin{abstract}
Metallic nanoparticles (NPs) are suitable platforms for miniaturized biosensing based on their optical and magneto-optical properties. It is possible to enhance the sensitivity of specific kinds of NPs by exploiting their optical and magneto-optical properties under suitable external magnetic field modulation. Here, the magneto-optical properties of Fe-Ag core-shell ferromagnet-noble metal NPs have been investigated as a function of the incident light frequency. For $\mathrm{Fe}-\mathrm{Ag}$ NPs with a concentration ratio around 25:75, an optical absorption band centered at $3 \mathrm{eV}$ due to localized surface plasmon resonance (LSPR) excitation is observed. A strong enhancement of the Faraday rotation is also observed, greatly exceeding the value estimated for pure Fe NPs, also associated with the LSPR excitation. Our findings open up the possibility of highly sensitive miniaturized magneto-optically modulated biosensing. (C) 2010 American Institute of Physics.
\end{abstract}

[doi:10.1063/1.3355905]

\section{INTRODUCTION}

Development of highly sensitive biosensors for the diagnosis and monitoring of diseases, drug discovery, proteomics, and environmental detection of biological agents is an extremely significant problem. ${ }^{1}$ In addition to the needs for enhanced sensitivity, the development of large-scale biosensor arrays composed of highly miniaturized signal transducer elements that can enable the real-time, parallel monitoring of multiple species imposes stringent requirements for highthroughput screening applications. ${ }^{2}$ Much biosensor research has been devoted to the evaluation of various signal transduction methods including optical, piezoelectric, magnetic, micromechanical, amperometric, and mass spectrometric. Although each of these methods has its individual strengths and weaknesses, a strong case has been made that optical sensors, in particular those based on evanescent electromagnetic fields such as propagating surface plasmon polaritons (SPPs) in planar $\mathrm{Au}$ and $\mathrm{Ag}$ surfaces, are fast becoming a preferred method in many sensing applications. ${ }^{3}$ SPPs are essentially electromagnetic waves that are trapped on the interface of two media with permittivity of different sign, typically between a metal and a dielectric, due to their interaction with the free electrons of the metal. ${ }^{4}$ In addition, SPPs can also appear in appropriately designed metallic and metallodielectric structures as localized surface plasmon resonance (LSPR) that is excited when the incident photon frequency is resonant with the collective oscillation of the conduction electrons. As a consequence, noble metal nanoparticles (NPs) exhibit a UV-visible absorption band not present in the bulk metal, ${ }^{5,6}$ resonant Rayleigh scattering with an efficiency equivalent to that of $10^{6}$ fluorophors, ${ }^{7}$ and

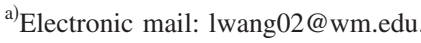

enhanced local electromagnetic fields near the surface of the nanoparticle. ${ }^{8}$ Plasmon resonances impart these nanostructures with unusual optical properties, such as strongly enhanced size-, shape-, and medium-dependent light absorption and have been employed in a wide range of applications including imaging, chemical, and biological sensing and probing with remarkable sensitivity. Thus, several research groups have explored optical biosensors ${ }^{9}$ based on the optical properties of noble metal NPs.

Although quite sensitive for many applications, most current biosensing schemes based on surface plasmon resonance and LSPR are "passive," i.e., they are based on changes in the optical properties of the gold-surface when a biological specimen to be detected is bound to it and surface plasmons are excited. Thus, to further enhance the sensitivity for more stringent applications, we have explored core-shell "magneto-optically active" plasmonic NPs. Here, the magneto-optical property can be varied under application of a modest external magnetic field hence enhancing the NPs inherent sensitivity by using field-modulated detection schemes that exploit their magneto-optical activity. Transition metals such as $\mathrm{Fe}, \mathrm{Ni}$, and $\mathrm{Co}$ alone exhibit magnetooptical effects accessible at relatively low fields, but their absorption coefficients are higher than those of $\mathrm{Au}$ or $\mathrm{Ag}$ and therefore their SPPs are considerably damped, but when combined with these noble metals their magneto-optical activity is enhanced due to the large electromagnetic fields that arise in the composite nanostructure when sharp SPP is excited in the noble metal. ${ }^{10-12}$ In fact, recent reports have indicated that the combination of noble metal $(\mathrm{Au})$ and magnetic materials (ferrite) in core-shell magnetic NPs exhibit remarkable magneto-optical effects, ${ }^{13}$ suggesting the possibility of implementing magnetoplasmonic materials to enhance sensitivity in biodetection. In the present case, we re- 
port on enhancement of the magneto-optical activity observed in core-shell $\mathrm{Fe}-\mathrm{Ag}$ magnetic NPs due to excitation of LSPR. The spectral absorbance and the magneto-optical Faraday rotation of the polarization of light through a magnetized medium composed of the core-shell Fe-Ag NPs suspended in a suitable liquid between glass slides was observed in transmission geometry.

\section{EXPERIMENTAL}

Core shell $\mathrm{Fe}-\mathrm{Ag}$ magnetic NPs were synthesized by aqueous reduction using sodium borohydride. The synthesis first included reduction of iron nanoparticles and was followed by the addition of silver nitrate. The iron served as a nucleation site for the reduction of silver, creating a coreshell nanoparticle. The particles were washed with ethanol several times and magnetically separated using a rare-earth magnet. X-ray powder diffraction revealed a mix phase system with a body centered cubic iron and face centered cubic silver. ${ }^{14}$ Transmission electron micrographs (TEMs) were taken on a JEOL JEM-1230 at $150 \mathrm{kV}$ with a Gatan Ultra Scan 4000 SP $4 \mathrm{Kx} 4 \mathrm{~K}$ charge coupled device camera to determine the size, dispersion, and morphology of the coated particles. The magnetization reversal was investigated by measuring hysteresis loops at room temperature (RT) on a Lakeshore model 7300 vibrating sample magnetometer. The sample was placed in a gelcap where the background was negligible compared to the sample signal.

For the optical and magneto-optical studies, the magnetic nanoparticles were suspended between two glass slides using index-matching oil with $\mathrm{n}=1.5018$. Absorption and Faraday rotation measurements were carried out in the spectral range $1.4-3.5 \mathrm{eV}$. The light incident beam was linearly polarized by a Glan Thompson polarizer (extinction ratio 100 000:1) and then transmitted through the sample. The sample was placed in the gap of an electromagnet with hollow polar pieces, allowing applied magnetic fields upto $1 \mathrm{~T}$, that were high enough to magnetically saturate the NPs. The transmitted light was analyzed by a polarizer positioned at $45^{\circ}$ with respect to the incident polarization. The intensity variations were detected using a Si photodetector and lock-in amplifier techniques.

\section{RESULTS AND DISCUSSION}

TEM was performed to determine the size distribution and morphology of the iron core Fe-Ag NPs. Figure 1(a) shows a TEM image corresponding to core-shell NPs with a $\mathrm{Fe}-\mathrm{Ag}$ concentration ratio of 75:25. Figure 1(b) shows a high resolution TEM image of the $\mathrm{Fe}-\mathrm{Ag}$ NPs illustrating their core-shell structure with body centered cubic iron core and face centered cubic silver shell with an average total particle size of $15 \mathrm{~nm}$ (core diameter $13.6 \mathrm{~nm}$ and shell thickness of $1.4 \mathrm{~nm}$ ). It is worth noticing that the nanoparticle size was found to be independent of the $\mathrm{Fe}-\mathrm{Ag}$ ratio. The NPs investigated for magnetoplasmonic effects have a $\mathrm{Fe}-\mathrm{Ag}$ concentration ratio of 25:75 (core diameter $9.4 \mathrm{~nm}$ and shell thickness of $5.6 \mathrm{~nm}$ ) since preliminary simulations showed that maximum Faraday rotation is expected around this concentration ratio.
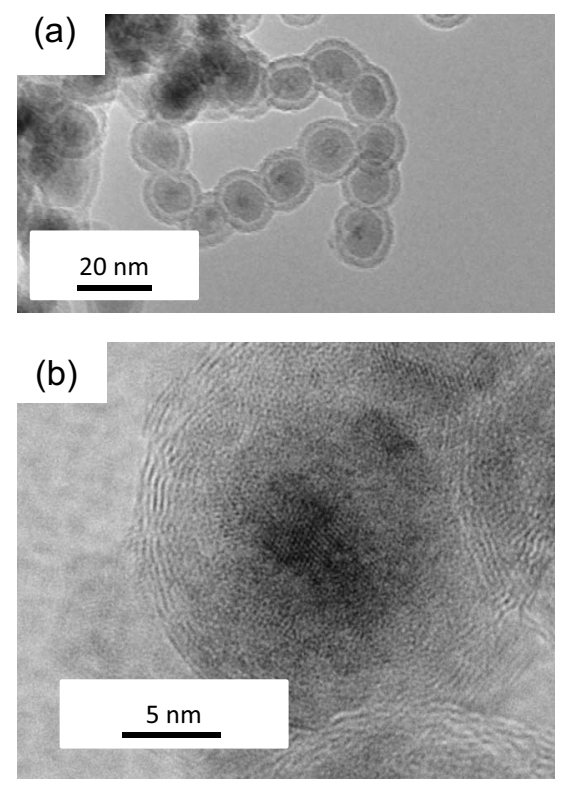

FIG. 1. (a) TEM image of Fe-Ag core-shell NPs synthesized by aqueous reduction. (b) High resolution TEM image of a $\mathrm{Fe}-\mathrm{Ag}$ nanoparticle, which illustrates the core-shell structure of the NPs with an average particle size of $15 \mathrm{~nm}$.

The magnetic properties were studied by measuring hysteresis loops at RT. As shown in Fig. 2, the NPs show characteristic superparamagnetic behavior with very low coercivity and high saturation fields around $5 \mathrm{kOe}$ due to the fact that their volume is well below the critical volume and corresponding superparamagnetic field, which for Fe NPs corresponds to diameters around $26 \mathrm{~nm} .{ }^{15}$

The absorbance and Faraday rotation of the $\mathrm{Fe}-\mathrm{Ag}$ coreshell NPs with optimum $\mathrm{Fe}-\mathrm{Ag}$ concentration ratio of 25:75 suspended in index-matching oil with $\mathrm{n}=1.5018$ were measured in the spectral range from 1.4-3.5 eV. As shown in Fig. $3(\mathrm{a})$, the absorbance exhibits an absorption band centered at $3 \mathrm{eV}$, signature of LSPR excitation as shown in previous reports. ${ }^{13}$ Associated with such absorption band there is a maximum in the Faraday rotation [Fig. 3(a) right]. Simulations of the effective dielectric tensors of the dispersed NPs films were performed using a variation in the MaxwellGarnett effective medium approximation presented by $A b e^{16,17}$ for composite core-shell systems in order to understand the observed behavior. For this study the bulk Fe and

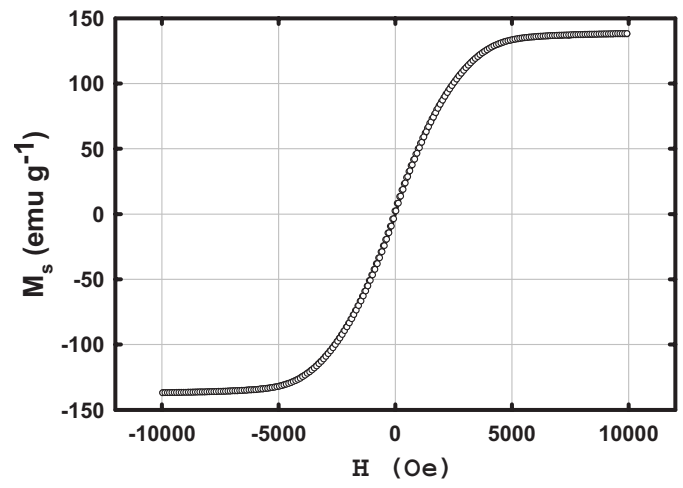

FIG. 2. Hysteresis loop for core-shell Fe-Ag NPs showing their superparamagnetic behavior at RT, with saturation fields of approximately $5 \mathrm{kOe}$. 


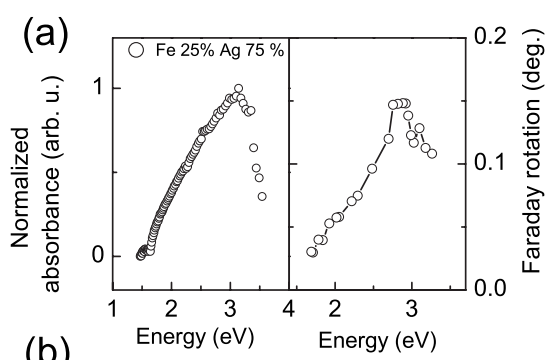

(b)

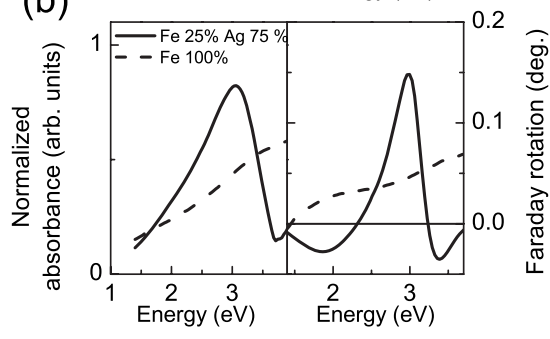

FIG. 3. (a) Experimental absorbance (left) and Faraday rotation (right) for $25 \% \mathrm{Fe}-75 \% \mathrm{Ag}$ NPs suspended in a liquid with $\mathrm{n}=1.5$. A maximum both in absorbance and Faraday rotation is found around $3 \mathrm{eV}$ due to LSPR excitation. (b) Simulations show for $25 \% \mathrm{Fe}-75 \% \mathrm{Ag}$ (continuous lines) and $100 \%$ Fe (dashed lines) NPs show the effect of LSPR on the optical and magnetooptical response of the system.

Ag optical and magneto-optical constants were used ${ }^{18}$ since the size of the NPs is well above that at which optical size effects have been described in NPs. ${ }^{19}$ Subsequently, the absorbance and Faraday rotation were calculated using a matrix transfer formalism, ${ }^{20,21}$ able to describe the behavior of light in multilayered systems. Figure 3(b) shows simulations using the cited formalism and considering a $25 \%$ concentration of NPs in the medium (continuous lines). As it can be observed, very good agreement is obtained between the simulation and the experimental data, confirming an absorption band centered at $3 \mathrm{eV}$ and an associated maximum in the Faraday rotation. For comparison, the response of Fe NPs with no coating and simulated under the same conditions is also shown in Fig. 3(b) with dashed lines. Due to the relatively high optical absorption of $\mathrm{Fe}$ in the UV and visible range, over-damped or no LSPR excitation is expected. It is worth noting that the experimental measurement of such Fe NPs with no coating cannot be achieved due to the high tendency of Fe to oxide, but their simulated response allows us to understand the effect of LSPR excitation on the optical and magneto-optical properties of core-shell Fe-Ag NPs. In fact, a broader and less intense absorption band is observed as in Fig. 3(b), indicating over-damped or no LSPR excitation. More interestingly, in spite of the fact that in this case the concentration $\mathrm{Fe}$ in the NPs is four times higher than in the 25:75 Fe- $\mathrm{Ag}$ NPs, a much smaller Faraday rotation is ob- served, thus evidencing a strong enhancement of the magneto-optical response for the $\mathrm{Fe}-\mathrm{Ag}$ core-shell NPs when the Ag content is higher.

\section{CONCLUSIONS}

We have investigated the microstructure, magnetism, optical, and magneto-optical properties of $\mathrm{Fe}-\mathrm{Ag}$ core-shell NPs. The NPs exhibit a mix phase system with a body centered cubic iron core and face centered cubic silver shell with average diameters around $15 \mathrm{~nm}$. Superparamagnetic behavior was observed due to their reduced dimensionality. For $\mathrm{Fe}-\mathrm{Ag}$ NPs with concentration ratio around 25:75 an optical absorption band centered at $3 \mathrm{eV}$ is observed due to LSPR excitation. Associated with such increased absorbance a strong enhancement of the Faraday rotation it is also observed, greatly exceeding the estimated value for pure $\mathrm{Fe}$ NPs.

${ }^{1}$ A. P. F. Turner, Science 290, 1315 (2000).

${ }^{2}$ A. J. Haes and R. P. Van Duyne, J. Am. Chem. Soc. 124, 10596 (2002).

${ }^{3}$ R. Wang, Biosens. Bioelectron. 20, 967 (2004).

${ }^{4}$ W. L. Barnes, A. Dereux, and T. W. Ebbesen, Nature (London) 424, 824 (2003).

${ }^{5}$ C. L. Haynes and R. P. Van Duyne, J. Phys. Chem. B 105, 5599 (2001).

${ }^{6}$ U. Kreibig, M. Gartz, A. Hilger, and H. Hovel, in Advances in Metal and Semiconductor Clusters, edited by M. A. Duncan (JAI, Stamford, CT, 1998), Vol. 4, pp 345-393.

${ }^{7}$ M.-Y. Ng and W.-C. Liu, Opt. Express 17, 5867 (2009); G. C. Schatz and R. P. Van Duyne, in Handbook of Vibrational Spectroscopy, edited by J. M. Chalmers and P. R. Griffiths (Wiley, New York, 2002), Vol. 1, pp 759-774.

${ }^{8}$ R. H. Ritchie, "Plasma losses by fast electrons in thin films," Phys. Rev. 106, 874 (1957)

${ }^{9}$ M. D. Malinsky, K. L. Kelly, G. C. Schatz, and R. P. Van Duyne, J. Am. Chem. Soc. 123, 1471 (2001); R. Elghanian, J. J. Storhoff, R. C. Mucic, R. L. Letsinger, and C. A. Mirkin, Science 277, 1078 (1997); C. A. Mirkin, R. L. Letsinger, R. C. Mucic, and J. J. Storhoff, Nature (London) 382, 607 (1996).

${ }^{10}$ J. B. González-Díaz, A. Garcia-Martin, G. Armelles, J. M. Garcia-Martin, C. Clavero, A. Cebollada, R. A. Lukaszew, J. R. Skuza, D. P. Kumah, and R. Clarke, Phys. Rev. B 76, 153402 (2007).

${ }^{11} \mathrm{G}$. Armelles, J. B. Gonzalez-Diaz, A. Garcia-Martin, J. M. Garcia-Martin, A. Cebollada, M. U. Gonzalez, S. Acimovic, J. Cesario, R. Quidant, and G. Badenes, Opt. Express 16, 16104 (2008).

${ }^{12}$ C. Hermann, V. A. Kosobukin, G. Lampel, J. Peretti, V. I. Safarov, and P. Bertrand, Phys. Rev. B 64, 235422 (2001).

${ }^{13}$ P. K. Jain, Y. H. Xiao, R. Walsworth, and A. E. Cohen, Nano Lett. 9, 1644 (2009).

${ }^{14}$ S. Naik and E. E. Carpenter, J. Appl. Phys. 103, 07A313 (2008).

${ }^{15}$ C. M. Boubeta, C. Clavero, J. M. Garcia-Martin, G. Armelles, A. Cebollada, L. Balcells, J. L. Menendez, F. Peiro, A. Cornet, and M. F. Toney, Phys. Rev. B 71, 014407 (2005).

${ }^{16}$ M. Abe and J. Kuroda, J. Appl. Phys. 91, 7305 (2002).

${ }^{17}$ M. Abe and T. Suwa, Phys. Rev. B 70, 235103 (2004).

${ }^{18}$ E. D. Palik, Handbook of Optical Constants of Solids (Academic, Orlando, 1985).

${ }^{19}$ C. Clavero, A. Cebollada, G. Armelles, Y. Huttel, J. Arbiol, F. Peiro, and A. Cornet, Phys. Rev. B 72, 024441 (2005).

${ }^{20}$ M. Schubert, Phys. Rev. B 53, 4265 (1996).

${ }^{21}$ M. Schubert, T. E. Tiwald, and J. A. Woollam, Appl. Opt. 38, 177 (1999). 